\title{
LA SUBLIMACIÓN DE LA BELLEZA
}

The sublimation of beauty

\author{
SIXTO J. CASTRO \\ Universidad de Valladolid (España) \\ sixto@fyl.uva.es
}

Resumen

En el presente texto trato de mostrar el cambio que ha supuesto para el concepto de belleza poner en paralelo los conceptos de lo bello y lo sublime en el mundo moderno. Me centro de modo especial en la pérdida del potencial revelatorio adjudicado a la belleza por el pensamiento platónico y sus herederos y en la transferencia de esta capacidad al concepto de lo sublime de Kant en adelante, si bien reducida esta capacidad al espacio inmanente. Ese cambio tendrá una influencia radical en otra serie de conceptos básicos del arte, entre ellos, el de genio.

Palabras clave: bello, sublime, Platón, Kant.

\section{Abstract}

In this paper I try to show the change the concept of beauty has gone through when it has run parallel to the concept of the sublime in the modern world. I focus particularly on the loss of the revelatory potential attributed to the beauty by the Platonic thought and its heirs, and on the transfer of this capacity to the concept of the sublime from Kant onwards, although this capacity will be reduced to the immanent space. This change will have a radical influence on a series of basic concepts of art, among them, that of genius.

Key words: beautiful, sublime, Plato, Kant.

\section{INTRODUCCIÓN}

De más está decir que en nuestra época la belleza ya no es el valor estético por antonomasia, al menos en el arte. Hay muchos otros que dominan el discurso artístico, y la belleza, a pesar de ostentar aún un brillo especial entre los valores, parece tener un cierto carácter intempestivo, fuera del tiempo, fuera de nuestro tiempo artístico. Sin embargo, en el espacio de lo cotidiano, la belleza se persigue, se valora, se vende, con ella se trafica y se mercadea (Hamermesh, 2011). Se ha convertido en una mercancía carente de potencial simbólico y revelador. Para que hayan podido producirse estos cambios, han tenido que pasar muchas otras cosas en la historia del pensamiento, entre ellas su confrontación con otro de los grandes términos de la tradición estética occidental: lo sublime.

Richard Rorty (2001) sostiene que la respuesta a la pregunta de ¿qué es filosofía? es "una discusión informada y voluntaria sobre lo bello y lo sublime" (p. 51). Independientemente del desarrollo de esta idea por parte del filósofo norteamericano, es cierto que siguiendo el itinerario y la relación mutua de estos dos términos -si consideramos su independencia mutua en un primer momento, la comprensión de cada 
uno de ellos en términos del otro más tarde y, finalmente, el triunfo total de lo sublime en el discurso estético (y no solo en el estético)- salen a la luz ciertas relaciones de fuerza fundamentales que han constituido la filosofía occidental en los últimos siglos. La relación dialéctica de estos dos términos se da en el marco de otros cambios filosóficos de no poca importancia, y, hasta cierto punto puede incluso hacerlos posibles.

\section{AUGE Y CAÍDA DE LA BELLEZA: LO BELLO Y ALGO MÁS}

"Lo bello es difícil", afirmaba Platón en el Hipias mayor, justo lo contrario de lo que sostiene Santayana (1969) en El sentido de la belleza, en cuya penúltima página afirma que "la belleza es, de todas las cosas, la que menos necesita de explicación" (p. 240). Llegar a esta afirmación, sin embargo, le ha costado 200 páginas. Es lógico que cualquier conclusión relativa a la belleza muestre este carácter dual, pues "belleza" es un término ambiguo. Por un lado, designa un valor o propiedad estética positiva: lo bello es lo que es, desde un sentido, estético excelente; por otro lado, la belleza es un tipo particular de valor o propiedad estética (algo armónico, placentero) que se opone, por ejemplo, a lo sublime o lo feo (Roche, 2013, p. 327). El primer sentido domina la reflexión tradicional, en la que no hay nada que se enfrente a lo bello. Todo lo que es, es bello hasta cierto punto según su propia naturaleza. La belleza es el ideal de lo que las cosas deberían ser, y la aparente ausencia de belleza, incluso cuando la llamamos fealdad, es en realidad una falta del único valor positivo que es la belleza, el que "por defecto" está presente en la realidad. Si el mundo fuese perfecto, sería un mundo bello. Por el contrario, en el pensamiento moderno, la belleza abandona su lugar privilegiado y compite con muchas otras propiedades estéticas. Esta depreciación del concepto tiene mucho que ver con la pesada carga metafísica asociada con el concepto. La crisis de la belleza en el discurso filosófico y artístico va de la mano también de la crisis de la metafísica misma.

Entre los antiguos griegos, la idea de belleza era inseparable de la idea de bondad, de la de verdad y de la idea de lo divino ${ }^{1}$. Se ve con claridad en el desarrollo del concepto que lleva a cabo Platón (1987, 1989, 1988a, 1988b, 2002a, 2002b, 2011) en Banquete, República, Hipias Mayor, y otros textos, en los que también indaga qué es lo que constituye esta realidad misteriosa, en qué términos puede describirse. Su preferencia son las matemáticas: la forma (Cratilo 493c, Eutidemo 301a, Leyes 655c, Fedón 65d, 75d, 100b, Fedro 254b, Parménides 130b, Filebo 15a, República 476b, 493e, 507b), la proporción y la unidad (Filebo 64e, 66b, Político 284b, Timeo 87c-d), y todas aquellas relaciones matemáticas que son parte del reino inteligible. Pero en Platón las matemáticas no son todo lo que es la belleza. Son lo que, en términos contemporáneos, llamaríamos su base subveniente, es decir, aquello que la funda "materialmente" (si así pueden

\footnotetext{
${ }^{1}$ Para algunas de las referencias históricas que siguen me baso en Ross (1998) y en Reschke (2003).

90 | Alpha № 53 (Diciembre 2021) PÁGs. 89-100. ISSN 07 16-4254
} 
entenderse las matemáticas), pero a lo que no puede reducirse. En apariencia la belleza no se da sin una base matemática, pero no se identifica con ese fundamento matemático. En Platón, la belleza es un concepto denso que incorpora elementos descriptivos (de raigambre matemática) y elementos evaluativos. Por eso, no parece que ninguna descripción sea del todo adecuada sin atender a ese elemento valorativo irreductible a la descripción.

Para Platón, la belleza es una forma, una idea de la que participan las realidades que llamamos bellas, es decir, todas las realidades. Para él, la belleza es una manifestación o, mejor, una presencia del mundo inteligible en el mundo sensible, de la que no es posible dudar. La duda al respecto no aparecerá en la historia del pensamiento hasta la modernidad, donde la belleza empezará a ser vista como engañosa. Pero para Platón no cabe la posibilidad de engañarnos. La belleza tiene un carácter revelatorio de lo inteligible en lo sensible: mediante las bellezas sensibles podemos llegar a la belleza inteligible, que es su origen, siguiendo la vía erótica, que realiza el anhelo del amante. Belleza y erotismo forman la unidad platónica peculiar que da a la "experiencia estética" platónica ese carácter exaltado, que contrasta con la concepción moderna de la misma. Plotino (1988, 2001), Agustín, y el Pseudo-Dionisio continúan esta idea platónica de que en la belleza aparece siempre algo más que el objeto o la realidad que consideramos bella: se hace patente la Belleza, el origen, y este elemento anamnético -como Platón, Plotino infiere que cuando el alma es atraída por la belleza de los cuerpos percibe el recuerdo de algo semejante a sí misma, más elevado, en esos cuerpos- conlleva en sí el placer de la contemplación de la idea de belleza. La tradición medieval desarrolla esencialmente esta idea bajo un tinte metafísico o teológico, con la diferencia de que, si en el mundo platónico toda belleza procede de la idea de belleza, en el mundo cristiano esa Belleza es ahora Dios mismo, pulchritudo tam antiqua et tam nova. Si para los platónicos toda belleza revela en sí la Belleza (idea), una vez que el platonismo se cristianiza, toda belleza será resplandor de la Belleza que ahora es Dios mismo. Esta idea queda bien expresada en $D e$ divinis nominibus, de Pseudo-Dionisio, para quien el predicado "bello" es uno de los nombres de Dios, que es quien confiere la belleza que es él mismo a las cosas, según la naturaleza de cada una (pp. 301-302). La belleza de las cosas es vestigio de la Belleza que es Dios, origen. Por eso la belleza tiene un potencial simbólico único y por esta razón las conformaciones artísticas van a ser un recurso religioso privilegiado, como se mostrará en las diversas querellas iconoclastas que ha habido a lo largo de la historia, de modo singular en las del siglo VIII.

La concepción de la belleza ligada a la armonía y considerada una propiedad de lo real -lo más real de lo real-, dotada de ese carácter revelador del orden subyacente a lo visible se quiebra con la modernidad. Una vez que se comience a sospechar de la idea misma de "orden" como un elemento constitutivo real del universo, el concepto tradicional de belleza va a entrar en crisis. Quizá el orden, como pensaba Hume (2004) en sus Diálogos 
sobre la religión natural, sea solo temporal, aparente, impuesto o se haya dado solo en una parte del universo, mientras que el resto es desordenado. Una vez que no podamos hablar de orden, proporción, y la noción de completitud o integridad deje de ser aplicable, el concepto de belleza acabará por volverse inútil o inutilizable y habrá que buscar otros que nos permitan relacionarnos estéticamente con la realidad a un nivel también profundo, lo que traerá como consecuencia la resemantización del término "belleza".

Este cambio no es solo una sustitución de algunos conceptos por otros. Lleva aparejada una nueva cosmovisión. Una vez que la reflexión estética cae bajo el paradigma del juicio del gusto, pasamos de considerar la belleza como la aparición que testimonia un origen a verla como una apariencia carente de arraigo en lo real. Mientras que la belleza, en la consideración tradicional, nos habla de lo real -nos dice algo de lo real- y de nosotros como parte de esto real, el juicio del gusto moderno solo nos puede decir algo acerca del individuo que juzga, en cierto modo alienado de lo real, y, en última instancia, respecto de nosotros mismos. Esto sucede en un mundo que, desde Descartes en adelante, se ha vuelto cuestionable como fuente epistémica de certeza y confuso en todo lo relacionado con la belleza. En el caso de Hume, la realidad fundamental ya no es la belleza, sino el gusto que, como se constata, difiere de persona a persona. Ahora el discurso de la belleza ya no se centra en el objeto, sino en el sentimiento del sujeto. La belleza ya no es una cualidad de las cosas mismas, sino que existe solo en la mente que la contempla. El observador se convierte en fundamental e incluso empieza a ocupar el espacio central en los cuadros que antes retrataban lo real. Este ataque a la realidad de la belleza provoca las protestas de muchos autores, de modo señalado las de Berkeley (1990):

\begin{abstract}
"iQué placer tan sincero el contemplar las bellezas naturales de la tierra!... ¿Qué tratamiento merecen, entonces, esos filósofos que privan de toda realidad a esas nobles y deliciosas escenas? ¿Cómo pueden sostenerse en consideración esos principios que nos llevan a pensar que toda la belleza visible de la creación es un falso brillo imaginario?” (Berkeley, 1990, pp. 135-137).
\end{abstract}

Lo nuevo no es que la belleza se considere subjetiva -idea que se puede rastrear en Grecia, aunque no fuese la dominante: ya desde Vitrubio se distingue entre simetría y euritmia, entendiendo esta última como la adecuación de las proporciones objetivas a las exigencias subjetivas de la visión-, sino que se establezca una división entre el conocimiento del mundo natural, que se considera objetivo, y la belleza y la bondad, que se reducen al espacio de lo subjetivo. Para tratar de encontrar una cierta norma frente a esta disolución de la belleza en la subjetividad, Hume somete el gusto a la consideración de los jueces verdaderos, personas libres de los muchos defectos que impiden a los demás emitir un juicio de gusto auténtico: "Los hombres que posean buen gusto unido a delicadeza de sentimiento, perfeccionado por la práctica y la comparación y libres de todo 
prejuicio. El veredicto unánime de esos jueces, donde quiera que estén, es la verdadera norma del gusto y belleza" (Hume, 2008, p. 261).

La resolución del juicio de gusto se deja en manos de esta comunidad de jueces ideales, libres de todo defecto, que se convierten en la norma del gusto y de la belleza: la norma "natural" del gusto resulta ser del todo artificial, una suerte de institución de jueces ideales que carecen de los defectos que nos caracterizan a la mayoría de nosotros cuando juzgamos y que, de hecho, carece de contenido fáctico más allá de la consagración de los modelos que constituyen el "mundo del arte" occidental frente al cual los jueces prueban sus capacidades (Castro, 2012).

La belleza, pues, ya no se considera parte del mundo objetivo; está en el ojo del espectador $\mathrm{y}$, como afirma Hume, cada espectador puede ver una belleza diferente, por eso hay que acudir a esa estructura de los jueces verdaderos o ideales para instaurar un criterio de certeza estética en un mundo que, desde ahora, rehúye toda posibilidad de que la belleza se considere real en sí misma. Para Hume (2008) no hay nada que podamos obtener de un análisis científico y llamarlo belleza. La percepción por sí sola no puede dar una explicación de la belleza en términos de propiedades primarias. En definitiva, la belleza parece no estar en los objetos. De ser lo más real de lo real, la belleza pasa a ser lo más opinable y sin sustancia alguna sobre la que debatir. A diferencia de lo que sucede en el ámbito ontológico en la modernidad, según el análisis heideggeriano (2010, p. 72), donde lo real se sustituye por lo objetivo, aquí es lo subjetivo lo que ocupa el lugar de lo real estético; pero en paralelo con lo que sucede en el ámbito epistémico, donde la verdad se sustituye por la certeza, aquí es la belleza real la que se sustituye por la certeza estética de los jueces ideales, que acaban cayendo en un razonamiento circular. En todo caso, la reducción de la belleza a un sentimiento (o de todo lo que se puede decir de la belleza a un estado de ánimo placentero) supone la ruptura de su carácter universal. El sujeto estético delineado por la filosofía moderna no puede universalizar. Solo puede hablar de sí mismo y, en último término, remitirse a esa comunidad de jueces ideales para justificar su juicio de manera intersubjetiva, pero en ningún caso fundado en realidad alguna que vaya más allá de esa comunidad circular. Lo que hasta entonces había sido una relación inmediata con lo bello, se convierte ahora en un juicio de gusto que la mayoría de las veces es erróneo y que cede su justificación al cuerpo extraño de los jueces.

Kant tratará todavía de salvaguardar el carácter universal de la belleza mediante su artificio de la "universalidad subjetiva", al considerar que el juicio de gusto se origina en el sentimiento de placer generado por el libre juego de la imaginación y el entendimiento, facultades que habitualmente operan para dar lugar al conocimiento, pero que, en el caso del juicio estético, no logran un conocimiento conceptual, sino que se mantienen en ese juego libre que combina la libertad de la imaginación y la legalidad del entendimiento. En la medida en que postulamos que ese mismo proceso acontece en todos los sujetos de la misma manera, podemos hablar de la universalidad de la belleza, 
pero subjetiva, porque no se apoya en un concepto, sino en un sentimiento. Esta aparente cuadratura del círculo pretende salvar para la belleza ese carácter de universalidad que $a$ priori consideramos que tiene, pero, al no poder fundarlo en el objeto (recordemos a Hume), hemos de fundarlo en el sujeto y en sus operaciones. Toda la carga metafísica que la belleza había arrastrado desaparece en Kant. Su espacio es el de las apariencias.

En la Crítica del Juicio, Kant (1977) define la belleza a partir del gusto en cuatro momentos negativos: 1) el gusto es satisfacción desinteresada; lo bello es el objeto de tal satisfacción; 2) lo bello place universalmente sin requerir un concepto; 3) lo bello se juzga a partir la forma de la finalidad de un objeto sin la representación de un fin; 4) lo bello es el objeto de una satisfacción necesaria independiente de un concepto. En otras palabras, la belleza no cae bajo concepto alguno, científico o ético; place desinteresadamente, sin finalidad y place (o mejor dicho, debería placer) en forma universal. Esta es la raíz de la postulada universalidad subjetiva del juicio de gusto. Usando un ejemplo wittgensteiniano, Scruton trata de explicar la posición de Kant. Cuando un carpintero elige, de entre los posibles marcos de una puerta, todos los que cumplen su función, elige tal o cual porque le parece el adecuado en virtud de su apariencia, liberándose así del razonamiento instrumental, eligiendo un objeto que ya no es medio para un fin, sino fin en sí mismo. Una vez cubiertas las cuestiones de función y utilidad, lo que queda es la apariencia. Y este interés en la apariencia corresponde a dos de las condiciones que Kant señala para lo estético: está ligado a la experiencia sensible y es desinteresado, pues surge solo cuando nuestros intereses prácticos ya se han cumplido o se han dejado de lado. Querer algo por su belleza implica abstraer de su función, y hasta de su misma existencia. Por eso podemos decir, con Scruton (2009), que "llamamos a algo bello cuando obtenemos placer de contemplarlo como un objeto individual, por sí mismo, y en su forma presentada" (p. 26). Esto lo desarraiga por completo de cualquier espacio que no sea el meramente fruitivo, que es la acusación fundamental que Gadamer lanza a la teorización kantiana. Para Gadamer (1996), en la Crítica del Juicio, Kant renuncia a que haya algún conocimiento del objeto cuando se dice de él que es bello y concluye que ese juicio solo atañe a la relación del objeto con nuestras facultades cognoscitivas en general (p. 63). Además, en Kant la belleza queda reducida al estatus de símbolo de la moralidad. La relación simbólica ya no es real, sino solo pragmática: representamos la bondad mediante la belleza, pero la bondad ya no se presenta en la belleza, como sucedía en el mundo platónico. Por último, en Kant comienza también el proceso de disolución de la belleza a favor de lo sublime. La belleza adquiere ahora los rasgos de orden, perfección y forma, frente a lo sublime, que asumirá los caracteres de lo que sobrepasa el orden, el fin y la forma ${ }^{2}$.

\footnotetext{
${ }^{2}$ Lo narrado hasta ahora puede tomarse como índice de un cambio. La belleza, para Tomás de Aquino, place a quien la ve (Summa Theologiae, q.5, a.4 ad 1), en último término a todos los que la ven, como el bien, ya que 94 | AlPha № 53 (Diciembre 2021) PÁGS. 89-100. ISSN 07 16-4254
} 
Tras Kant, la reflexión acerca de la belleza sigue presente, por ejemplo, en la obra de Hegel, que la vincula necesaria y en exclusiva al arte, pero esta es algo del pasado, de modo que la belleza va a ser desalojada también como categoría estética. El arte se hará sin la referencia a la belleza y el aspecto puramente filosófico del arte toma su lugar, convirtiendo al arte en algo más semántico que estético.

En el panorama filosófico esbozado quedamos, en cierto modo, huérfanos de belleza. El mismo proceso de despliegue del espíritu humano parece volverla innecesaria. Si sirvió para que el espíritu se autocomunicase, ahora ya parece que no es útil para este fin. Dentro del mundo del arte, el término "belleza" va a ser cada vez menos utilizado. Otras categorías estéticas ocasionales ocupan su lugar: lo siniestro (das Umheimliche), lo abyecto, lo camp, lo kitsch, lo trash, etc. Todas ellas, sin embargo, beben, al menos en parte, de un concepto que sí tiene una importancia histórica grande: lo sublime, y la superación de lo bello por este tiene consecuencias no solo para la estética, sino para nuestra misma concepción de lo real.

\section{LO SUBLIME TOMA EL CONTROL}

Hoy usamos el término sublime cuando hablamos de agujeros negros, de un paisaje alpino, de una pintura de Mark Rothko, de una pieza de piano de Stockhausen, de una obra arquitectónica de Frank Gehry, de una performance de Marina Abramovic, o de cualquier obra que nos desafíe emocional e intelectualmente. También lo aplicamos al ámbito cotidiano sin demasiadas precisiones. Sublime, según el diccionario de la RAE, significa "1. adj. Excelso, eminente, de elevación extraordinaria. U. m. en sent. fig. apl. a cosas morales o intelectuales. 2. adj. Dicho de una persona: Que cultiva algún arte o técnica con grandeza admirable (...). 3. adj. Ret. Dicho del estilo: Dotado de extremada nobleza, elegancia y gravedad". Este último es el sentido que tiene originalmente en la obra del Pseudo-Longino (Sobre lo sublime), autor del siglo I d. C., quien lo utiliza para referirse a un tipo de discurso que trata no de proporcionar placer o de ser útil, sino de lograr una intensa conmoción de la mente, un éxtasis que sorprende al alma y la mueve con la fuerza de la pasión, en contraposición a lo bello, que deleita y resulta de la armonía del discurso. El discurso sublime cae sobre nosotros, nos provoca una suerte de éxtasis y revela al alma su propia infinitud. Platón había observado este efecto en la poesía para desacreditarlo, pero el autor de Sobre lo sublime construye toda una poética respecto de la valoración de este pathos. Ahora se supone que la pasión es la fuente de la obra.

Boileau traduce este tratado en el siglo XVII, y ejerce una gran influencia en el siglo XVIII, donde las ideas sublimes se tratan de manera profusa en la obra de John

\footnotetext{
la belleza solo se distingue del bien sub specie rationis y el bien es lo que place a todo el mundo (est enim bonum quod omnia appetunt). Según Hume, la belleza solo place a los jueces verdaderos, los dotados de la capacidad de emitir un juicio de gusto puro. Finalmente, para Kant, la belleza solo le place a uno mismo (aunque debiera placer a todos).
} 
Dennis, Addison, Shaftesbury, Burke, Priestley, etc. En el siglo XVIII lo sublime se refiere a una clase muy limitada de objetos o nociones, en la que se puede incluir cualquier objeto que se considere que excite una de estas pasiones, sentimientos o emociones: elevación, transporte, entusiasmo, exaltación, estupefacción, éxtasis, terror entusiasta, horror delicioso, asombro agradable, admiración apasionada, temor indescriptible, complacencia inefable, entusiasmo sagrado, locura extática, rapto divino (Kirwan, 2005, p. 3). Así, la idea de sublimidad, que hoy consideramos típicamente estética, pertenecía en el siglo XVIII al campo de la filosofía moral, donde se estudiaban sobre todo las pasiones y los sentimientos. No se trataba tanto de la experiencia estética como de la experiencia en general. Por ejemplo, Kant (2008), en la sección III de sus Observaciones acerca del sentimiento de lo bello y de lo sublime, una obra que recuerda mucho a De lo sublime y lo bello de Burke, compara a los hombres (sublimes) con las mujeres (bellas), y se refiere a los diversos temperamentos (estados de ánimo) como más o menos adecuados para comprender lo bello o lo sublime. Es el melancólico quien tiene una sensibilidad especial para lo sublime.

El Pseudo-Longino no opuso hýpsos a kalós; los primeros en hacerlo fueron Addison y Burke, a mediados del siglo XVIII y con ello se dio un cambio en la misma concepción de lo bello, que ahora va a mutar su significado -al menos el que tiene en la filosofía griega y medieval- al ser contrapuesto a lo sublime. Al principio, lo sublime se entendía como un aspecto de lo bello, pero esta identificación no duró mucho. En De lo sublime y de lo bello, Burke (2005) señala que lo bello se origina en la armonía y el orden. Lo sublime, por el contrario, en la grandeza y el poder. Aquel en el amor, este en el miedo. Pero ambos nos elevan por encima de los pensamientos utilitarios que dominan nuestra vida cotidiana. A diferencia de lo bello, lo que caracteriza a lo sublime es un momento de disgusto seguido de un placer, y esto es lo que lo distingue del puro horror. Esto se debe bien a la sensación de seguridad frente a lo que nos amenaza, bien al hecho de que lo sublime refleja la grandeza interna del alma (esta última justificación está en especial presente en Longino).

El contraste entre lo bello y lo sublime adquiere su forma definitiva cuando Kant (1977), en la Crítica del Juicio, lo vincula a la diferencia entre lo finito y lo infinito, lo condicionado y lo incondicionado, entre entendimiento y razón. En el mundo kantiano, cuando juzgamos algo como bello experimentamos la adaptación de la mente y la realidad, mientras que cuando lo juzgamos sublime tenemos la experiencia de los límites de nuestras capacidades cognitivas y nos damos cuenta de que no nos es posible conocer el mundo como una totalidad infinita. Mientras que la experiencia de la belleza apuntala al sujeto en lo real, lo hace consciente de que el mundo al que tiene acceso cognitivo no le es ajeno (ya que, como señalamos, en el juicio de lo bello intervienen imaginación y entendimiento, las mismas facultades que se ponen en movimiento para elaborar el conocimiento conceptual), la experiencia de lo sublime lo supera y lo sobrepasa, lo 96 | Alpha No 53 (Diciembre 2021) PÁgS. 89-100. ISSN 07 16-4254 
descentra, le hace tomar conciencia de su finitud, de su insignificancia en el universo, y marca un límite a su capacidad de conocer. Juzgar que la naturaleza es bella, en la filosofía kantiana, es sentir una satisfacción que Susan Neiman (2015) interpreta en estos términos cuasiteológicos: "Si yo hubiera creado el mundo, lo habría hecho precisamente asî"; implica tener una idea de perfección. Juzgar que la naturaleza es sublime es ser consciente de que hay algo que sobrepasa nuestras capacidades: "por muy grandes que imagine mis poderes creadores, nunca serán suficientes para hacer eso" (p. 83). Cuando lo sublime se hace cargo, desaparece la idea misma de perfección, de orden, de cosmos. Toda revelación posible ha de ser ahora mediada por las ideas de la razón -lo pensable, no lo cognoscible-, que vienen en ayuda del sujeto y lo hacen consciente de su naturaleza suprasensible -moral-. El cambio es fundamental: lo suprasensible, lo nouménico, ya no será revelado por lo bello, sino por lo sublime, lo que trae consigo una depreciación de la belleza en su función revelatoria. La misma consideración de la naturaleza como sublime también implica un cambio en la forma de concebirla. Ya no se puede pensar en un sentido empírico (como en la Crítica de la razón pura), sino sobre todo como un exceso que no es reducible a lo empírico (como en la Crítica del Juicio): las magníficas montañas, las poderosas cascadas, los grandes desiertos, el cielo estrellado sobre nosotros señalan ese exceso que, al principio, nos hace sentir insignificantes, pero pronto, gracias a la ayuda de las ideas de la razón, causa una elevación que revela algo que está más allá de la representación, algo que pertenece al reino de lo suprasensible: el destino infinito del ser humano como ser moral, la revelación del hecho de que el ser humano es más que su naturaleza sensible.

Lo sublime, pues, toma el lugar de la naturaleza bella en la revelación de lo suprasensible, pero esta revelación solo puede tener lugar en un espacio inmanente. La posibilidad de ascender a lo trascendente propia de la belleza le está vedada a la experiencia de lo sublime. La conversión de lo sublime en categoría estética principal supone la inversión del ascenso platónico: ya no partimos de las realidades sensibles para llegar al mundo inteligible que es su fundamento, sino que penetramos de manera directa en la profundidad del sujeto, donde horadamos cada vez más sin la esperanza de hallar fundamento alguno. Ya no buscamos el "brillo" de la belleza, sino la oscuridad de lo sublime, de la que dependen buena parte de los conceptos clave del pensamiento moderno y contemporáneo: la voluntad schopenhaueriana, lo dionisíaco nietzscheano, lo inconsciente freudiano... hasta llegar, probablemente, al gen egoísta de Dawkins, una realidad que se postula como fundante, siempre esquiva, inaccesible y, sobre todo, oscura desde todos los puntos de vista.

Lo sublime adquiere de este modo el carácter de la presentación de lo no presentable. Se configura, según Milbank (2004), como "esa cualidad dentro de la representación que excede la posibilidad de representación" (p. 212) y así adquiere, en palabras de Lyotard (1987), el carácter de la presentación de lo inefable, de "lo impresentable en la presentación 
misma, aquello que niega ello mismo el consuelo de las buenas formas"(p. 25), una realidad que alude a algo pensable que no puede ser representado.

Ya no estamos, pues, en el mundo estético platónico, ni en el neoplatónico o en sus herederos cristianos. Podemos comprobar cómo el concepto de belleza ha cambiado y ha perdido su sustancia cuando comparamos las exaltadas afirmaciones platónicas en Banquete respecto del potencial revelador, encantador, afirmador de nuestra experiencia de lo real que tiene la belleza, con las comedidas y sospechosas reflexiones de Hume, para el que la belleza queda reservada a un pequeño número de escogidos que tienen que realizar complejas operaciones de análisis para certificar que, en efecto, lo que han contemplado puede ser considerado bello, a pesar de que sea solo una experiencia puramente subjetiva ${ }^{3}$. Si lo bello tradicional se refiere a un origen trascendente que muestra en su mismo aparecer, lo bello y lo sublime modernos permiten evitar cualquier tránsito hacia ese origen, ya que no hay otro origen más allá del sujeto. De modo especial, en el concepto de lo sublime hay un triunfo absoluto de la subjetividad inmanente en el pensamiento filosófico. El origen, todo origen, está en la acción o experiencia humana, no fuera. La canonización de lo sublime implica por fuerza un eclipse de la belleza "real" o "sólida". Ya no hay nada que pueda oponerse a la subjetividad libre y creadora, de modo semejante a como sucedía en la controversia religiosa nominalista y reformada, en la que no se aceptaba que existiesen esencias preexistentes que pudieran limitar la libertad omnímoda y la omnipotencia absoluta de Dios. El exceso romántico y postromántico de la subjetividad, que en parte es una secularización de la polémica teológica nominalista (Blumenberg, 2008), provoca que la interioridad infinita ya no se sienta en casa en el espacio de la belleza, que ahora, al haber sido contrapuesta a lo sublime, solo apunta a la forma definida, limitada y placentera, rompiendo con la tradición platónica de la belleza maníaca y la medieval de la belleza epifánica. Nada determinado puede oponerse a esta pretensión de infinito. Pero, en tanto que lo sublime implica una conciencia de un límite, nos hace al mismo tiempo, conscientes de lo que hay más allá de él. Esta será una idea clave para los románticos: la experiencia de lo sublime nos permite vernos en relación con una realidad absoluta.

Esto tiene como consecuencia que el artista, creador de belleza en la materia, según la concepción neoplatónica, pasa a ser sustituido por el genio. En el mundo premoderno, el artista crea belleza al introducir la forma de belleza en la materia -una forma que no está en él, sino de la que él participa, pero le preexiste-, de ahí que el arte adquiera un potencial simbólico: permite al contemplador remontarse a la Belleza

\footnotetext{
3 "Hagamos una experiencia e intentemos probar la intensidad de la belleza o la deformidad de algo: debemos escoger con cuidado el tiempo y lugar propicios, y dirigir la imaginación hacia la situación y en la disposición adecuadas. Debemos lograr una perfecta serenidad de la mente, poner en orden los pensamientos, prestar una debida atención al objeto; y si en cualquier de esas circunstancias falta, nuestro experimento será fallido y no podremos juzgar la belleza sincera y universal" (Hume, 2008, p. 252).
}

98 | AlPHA № 53 (Diciembre 2021) PÁGS. 89-100. ISSN 07 16-4254 
(platónica o divina) que es el origen de esa belleza. En el mundo moderno, el genio mismo deviene origen y no remite a nada más allá de su propia subjetividad. Por eso no se somete a regla alguna, y se vuelve él mismo origen. Cada una de sus obras es un ejemplar que no puede juzgarse frente a modelo alguno. Eso que en el mundo platónico había suscitado la condena (Ion, República) se vuelve el ideal del artista. El genio devendrá en nuevo sacerdote y lo sublime será el nuevo espacio espiritual en un mundo, el dieciochesco, en el que las seguridades proporcionadas por la religión han empezado a debilitarse.

\section{CONCLUSIÓN}

Lo aquí expuesto ha tratado de mostrar que el cambio de conceptos estéticos no es una cuestión menor o marginal, sino que lleva consigo un cambio de cosmovisión que afecta a múltiples consideraciones filosóficas. La belleza ha desaparecido del espacio filosófico fundamentalmente porque el espacio de apertura a lo pensable pero no conceptualizable ha sido ocupado por lo sublime. Pero la belleza ha migrado de la filosofía y el arte a otros espacios. Ha perdido su B mayúscula, su capacidad revelatoria, porque el mundo en el que se muestra no admite revelaciones, pero, manteniendo algunos elementos de la concepción tradicional, ha colonizado el mundo en el que vivimos, al servicio, en ocasiones, de causas nada bellas.

\section{OBRAS CITADAS}

Berkeley, George (1990). Tres diálogos entre Hilas y Filonús. Alianza. Traducción de Concha Cogolludo.

Blumenberg, Hans (2008). La legitimación de edad moderna. Pre-textos. Traducción de Pedro Madrigal.

Burke, Edmund (2005). De lo sublime y de lo bello. Alianza. Traducción de Menene Gras.

Castro, Sixto J. (2012). ¿Qué hay de malo en 'No nos gusta?, Estudios Filosóficos 178: 527-544.

De Aquino Tomás (2009-2010). Suma teológica. BAC. Traducción de los dominicos de España.

Demetrio-Longino (1979). Sobre el estilo. Sobre lo sublime. Gredos. Traducción de José García López.

Gadamer, Hans-Georg (1996). Estética y Hermenéutica. Tecnos. Traducción de Antonio Gómez Ramos.

Hamermesh, Daniel S. (2011). Beauty pays. Why attractive people are more successful. Princeton University Press.

Heiddeger, Martin (2010). La época de la imagen del mundo, en Caminos del bosque. Alianza. Traducción de Helena Cortés y Arturo Leyte.

Hume, David (2008). La norma del gusto, en Ensayos morales y literarios. Tecnos. Traducción de Estrella Trincado. 
— (2004). Diálogos sobre la religión natural. Tecnos. Traducción de Carmen García Trevijano.

Kant, Immanuel (2008). Observaciones acerca del sentimiento de lo bello y de lo sublime. Alianza. Traducción de Luis Jiménez Moreno.

_ (1977). Crítica del Juicio. Espasa-Calpe. Traducción de Manuel García Morente.

Kirwan, James (2005). Sublimity. Routledge.

Lyotard, Jean-François (1987). La posmodernidad (explicada a los niños). Gedisa. Traducción de Enrique Lynch.

Milbank, John (2004). Sublimity: The Modern Trascendent, en Schwartz, Regina (ed.), Transcendence: Philosophy, Literature and Theology Approach the Beyond. Routledge.

Neiman, Susan (2015). Evil in Modern Thought. Princeton University Press

Platón (2011). Filebo. Encuentro. Traducción de Ester Sánchez.

— (2002a). Cratilo. Trotta. Traducción de Atilano Domínguez.

— (2002b). Eutidemo. UNAM. Tradución de Osmanczik Smith.

— (1998). Fedón, Fedro. Alianza. Traducción de Luis Gil.

— (1989). El banquete. Alianza, Traducción de Fernando García.

—_ (1988a). La República. Gredos. Traducción de Conrado Eggers.

— (1988b). Las Leyes. Akal. Traducción de José Manuel Ramos.

— (1987). Parménides. Alianza. Traducción Guillermo R. de Echandía.

Plotino $(1988,2001)$. Enéadas. Gredos. Traducción de Jesús Igal.

Pseudo-Dionisio Areopagita (1990). Obras completas. BAC. Traducción de Teodoro H. Martín.

Reschke, Renate (2003). Schön/Schönheit, en Ästhetische Grundbegriffe. J. B. Metzler, Band 5: 390-436.

Roche, Mark W. (2013). The function of the ugly, en Hösle, Vittorio, The many faces of beauty. University of Notre Dame Press.

Rorty, Richard (2001). La belleza racional, lo sublime no discursivo y la comunidad de filósofas y filósofos. Logos. Anales del Seminario de Metafísica 3: 45-65. Traducción de Dámaso López.

Ross, Stephen D. (1998). Beauty: Conceptual and Historical Overview, en Michael Kelly (ed.), Encyclopedia of Aesthetics. Oxford University Press, vol. 1: 237-244.

Santayana, George (1969). El sentido de la belleza. Losada. Traducción de J. Rovira Armengol. Scruton, Roger (2009). Beauty. Oxford University Press. 